\title{
Dynamic deformable models for 3D MRI heart segmentation
}

\section{Leonid Zhukov, Zhaosheng Bao, Igor Gusikov, John Wood, David E. Breen}

Leonid Zhukov, Zhaosheng Bao, Igor Gusikov, John Wood, David E. Breen, "Dynamic deformable models for 3D MRI heart segmentation," Proc. SPIE 4684, Medical Imaging 2002: Image Processing, (9 May 2002); doi: $10.1117 / 12.467105$

SPIE. Event: Medical Imaging 2002, 2002, San Diego, California, United States 


\title{
Dynamic Deformable Models for 3D MRI Heart Segmentation
}

\author{
Leonid Zhukov ${ }^{a}$, Zhaosheng $\mathrm{Bao}^{a}$, Igor $\mathrm{Guskov}^{b}, \mathrm{John}_{\mathrm{Wood}^{c}}$ and David Breen ${ }^{a}$ \\ ${ }^{a}$ Department of Computer Science, California Institute of Technology, Pasadena, CA \\ ${ }^{b}$ Department of Electrical Engineering and Computer Science, \\ University of Michigan, Ann Arbor, MI \\ ${ }^{c}$ Childrens Hospital of Los Angeles, Los Angeles, CA
}

\begin{abstract}
Automated or semiautomated segmentation of medical images decreases interstudy variation, observer bias, and postprocessing time as well as providing clincally-relevant quantitative data. In this paper we present a new dynamic deformable modeling approach to 3D segmentation. It utilizes recently developed dynamic remeshing techniques and curvature estimation methods to produce high-quality meshes. The approach has been implemented in an interactive environment that allows a user to specify an initial model and identify key features in the data. These features act as hard constraints that the model must not pass through as it deforms. We have employed the method to perform semi-automatic segmentation of heart structures from cine MRI data.
\end{abstract}

Keywords: Deformable models, dynamic meshes, segmentation, heart MRI

\section{INTRODUCTION}

Cine magnetic resonance imaging has become the gold-standard for measurement of myocardial mass and function. Quantization of ventricular mass and function are important in myocardial diseases, such as coronary artery disease, myocarditis, and cardiomyopathy, as well as systemic diseases such as hypertension, diabetes, and cancer. In this regard, cardiac magnetic resonance imaging (MRI) has consistently demonstrated superior accuracy and reproducibility to 2D-echocardiography without the ionizing radiation needed by computed tomography. Typically, cross-section images at 10-12 anatomic levels are imaged at 16-32 equally spaced points throughout the cardiac cycle. Current state-of-the-art commercial tools use semi-automatic boundary detection to trace endocardial and epicardial boundaries at their maximum (end-diastolic) and minimum (end-systolic) frames. The traced contours are then combined to produce boundary representation of the volumes.

Unfortunately, in a clinical setting, postprocessing remains laborious and error-prone, despite commercial "computer-assisted" contour detection algorithms. At Childrens Hospital of Los Angeles, typical postprocessing times for left ventricular endocardial and epicardial boundaries is 20 minutes; combined left and right ventricular volumes take approximately 1 hour, despite only analyzing end-systolic and end-diastolic time-points. As a result, quantitative function analysis is reserved for selected indications, even though it is potentially clinically relevant in a much broader patient population. Thus the goal of our work is to develop a clinically useful, semi-automatic method to reduce postprocessing of cardiac cine-MRI images. Given the inherent difficulty that level and gradient techniques encounter near isocontrast boundaries, such as papillary muscle next to the ventricular free-wall, we developed a method that combines a small number of user-defined constraint points with a deformable model using some of the latest advances in mesh processing technology. The method allows a user to quickly extract a 3D model of heart structures by specifying key features in an MRI dataset and controlling a deformable model.

There has been significant research in the area of deformable models for medical image segmentation. Several excellent surveys of this work exist. ${ }^{1-3}$ Our approach builds on this work and is most closely related to Miller ${ }^{4}$

Further author information: (Send correspondence to L.Z) L.Z : Email:zhukov@cs.caltech.edu; J.B.: Email: jbao@cs.caltech.edu; I.G.: guskov@eecs.umich.edu; D.B. :Email: david@gg.caltech.edu 
and McInerney. ${ }^{5}$ These two approaches are similar in that they use an active deformable mesh to extract structures from medical image data.

In this paper we propose several significant modifications to these schemes. We employ a recently developed dynamic meshing algorithm ${ }^{6}$ that maintains an evenly sampled high-quality mesh during the deformation process and allows us to use a simple tessellated sphere to initialize our deformable model. This algorithm ensures that properties of the mesh, e.g. edge length, vertex valence, and aspect ratio, remain within user-specified values. High-quality meshes are essential when performing stable and reliable analysis and visualization. Our approach also utilizes a newly developed, accurate curvature metric for surface meshes ${ }^{7}$ to smooth the model during deformation. Curvature measures have been used for smoothing in level set models, ${ }^{8}$ and have been found to be quite effective. We considered using level set models ${ }^{9}$ for our segmentation task. Since their topological genus cannot be controlled (Level set models can split into several pieces and form holes), they were deemed unsuitable for our application, as well as too computationally expensive and complex.

We have implemented our deformable model within an interactive environment that allows a user to view the dataset with three orthogonal cutting planes. Additionally, the user can specify constraint points on individual slices to identify key features in the data that the model must pass through. The points act as hard boundary constraints. As the model deforms the points constrain the motion of any face that comes within a small distance to them. This is in contrast to other methods that use springs to impose soft constraints on deformable models. Having constraint points that limit motion, curvature metrics that enforce smoothing while being attracted to high constrast edges produces a deformable model that will expand out to the user-defined features and smoothly interpolate the regions in between.

The remainder of the paper details the methods used to implement our model and the segmentation results produced by it.

\section{METHOD}

\subsection{Data}

Cardiac images were collected using a 4 element phased-array torso coil on a 1.5 T CVi System (General Electric Medical Systems, Milwauke, WI) running CNV 3 system software. Breath-hold cine steady-state free precession gradient echo images (SSFP) were collected at the cardiac short axis plane. SSFP parameters were as follows: $40 \times 40 \mathrm{~cm}$ field of view (FOV), 128 phase encodes, 256 frequency encodes, 1 excitations (NEX), slice thickness 9 mm (short axis), TE $1.5 \mathrm{~ms}$, TR $3.1 \mathrm{~ms}, 16$ views per segment (VPS), 20 reconstructed cardiac phases, yielding true voxel resolution of $1.6 \mathrm{~mm}$ x $9 \mathrm{~mm}$ x $3.1 \mathrm{~mm}$. Images were Fourier interpolated by 2 in the phase dimension. 14 slices were collected in short axis dimension.

\subsection{Deformable Model}

The dynamic equation for the motion of a deformable model can be written as a set of second order ordinary differential equations for the vertex position:

$$
\mathbf{M} \frac{d^{2} \overline{\mathbf{P}}(\mathbf{r}, \mathbf{t})}{d t^{2}}+\mathbf{C} \frac{d \overline{\mathbf{P}}(\mathbf{r}, \mathbf{t})}{d t}+\mathbf{K} \overline{\mathbf{P}}(\mathbf{r}, \mathbf{t})=\overline{\mathbf{F}}(\mathbf{r}, \mathbf{t})
$$

where $\mathbf{M}$ is the mass matrix, $\mathbf{C}$ is a damping factor, $\mathbf{K}$ is a stifness matrix of the mesh structure and $\overline{\mathbf{P}}$ is a vector of all vertices. $\overline{\mathbf{F}}$ is a vector of external forces.

We are looking for a static solution and following ${ }^{5}$ we set $\mathbf{M}$ to zero, thus removing inertia. Then the model will come to rest when forces equilibrate. We can also substitute the stiffness matrix term with a force due to the curvature of the mesh (see section 2.4). This force should oppose the motion of the regions with high curvature, thus keeping the model smooth. After the renormalization the system of equations becomes

$$
\frac{d \mathbf{P}(\mathbf{r}, t)}{d t}-\beta \cdot \overline{\mathcal{K}} \mathbf{n}(\mathbf{P})=\mathbf{F}(\mathbf{P}) .
$$


The object grows due the to expansion force until it reaches the boundary, where the expansion force equals the curvature term and the gradient image force. Those elements that reach the vicinity of constraint points are given infinite masses and no longer move. Due to the stiffness of the mesh (guaranteed through the curvature term) the neighbors of the constrained elements have reduced mobility.

\subsection{Forces}

The external forces on the RHS of Eq.(2) consist of two terms: an image force and an expansion (balloon) force:

$$
\mathbf{F}=\mathbf{F}_{\text {image }}+\mathbf{F}_{\text {exp }}
$$

The image force is used to attract the model to significant 3D intensity edges (gradients). It is constructed from the image intensity as described in Gupta. ${ }^{10} G_{\sigma}$ denotes Gaussian smoothing filter with width $\sigma$.

$$
\mathbf{F}_{\text {image }}=-\alpha \cdot \nabla\left|\nabla\left(G_{\sigma} * I\right)\right|
$$

To compute the force at any point on the model from a discrete $I(i, j, k)$ image dataset, we use linear interpolation.

When the shape of the model is not close enough to the surface of the object, the image forces may not be able to attract it. To compensate for this, an inflating force is used to expand the surface towards the boundary. This force is oriented normal to the evolving surface and has a constant value.

$$
\mathbf{F}_{\text {exp }}=\gamma \cdot \mathbf{n}
$$

\subsection{Curvature Computations}

We use a differential geometry definition of the mean curvature

$$
\overline{\mathcal{K}} \mathbf{n}=1 / 2 \lim _{\operatorname{diam}(\mathcal{A}) \rightarrow 0} \frac{\nabla \mathcal{A}}{\mathcal{A}}
$$

where $\mathcal{A}$ is an infinitesimal area around a point on the surface, where we want to calculate the surface curvature. We find the curvature directly from the surface using the operator introduced in Desbrun, ${ }^{7}$ since it guarantees a zero tangential component. The discrete formulation of the above definition is

$$
\overline{\mathcal{K}} \mathbf{n}\left(\mathbf{P}_{i}\right)=-\frac{1}{4 A} \sum_{j \in N(i)}\left(\cot \alpha_{j}+\cot \beta_{j}\right)\left(\mathbf{P}_{j}-\mathbf{P}_{i}\right)
$$

where $\alpha_{j}$ and $\beta_{j}$ are the two angles opposite to the edge in the two triangles having the edge $e_{i j}$ in common. $A$ is the sum of the areas of the triangles having $\mathbf{P}_{i}$ as a common vertex. After normalization the equation (7) becomes

$$
\overline{\mathcal{K}} \mathbf{n}\left(\mathbf{P}_{i}\right)=-\frac{\sum_{j \in N(i)}\left(\cot \alpha_{j}+\cot \beta_{j}\right)\left(\mathbf{P}_{j}-\mathbf{P}_{i}\right)}{\sum_{j \in N(i)}\left(\cot \alpha_{j}+\cot \beta_{j}\right)} .
$$

\subsection{Dynamic Meshes}

An important part of our surface deformation routine is the procedure that ensures the quality of the evolving mesh, by controlling mesh vertex density and maintaining good aspect ratios in the individual triangles. This is important because the approximation properties of the curvature operator may suffer if it operates on poorly shaped triangles. Additionally, for image forces to be adequately represented, the surface sampling has to be sufficiently dense. Taking these factors into consideration, we have implemented the dynamic mesh approach presented by Kobbelt et al.. ${ }^{6}$ It guarantees both acceptable aspect ratios between the smallest edge and the largest edge (at most 1:2), as well as an average valence approaching 6 .

During the deformation, adaptive subdivision is invoked to ensure that adequate resolution is maintained for the model. Edges longer than predefined $\epsilon$ are removed by midpoint insertion. An edge collapse is performed 
when an edge is too short, less than epsilon/2 in this case. The endpoint with lower valence is collapsed into the end with higher valence and the neighbors from the two original vertices are combined. In order to prevent deformities, special care is taken to not collapse valence 3 vertices. Finally, edge swapping is performed periodically to optimize geometric properties and force the average valence to be as close to 6 as possible. For two neighboring triangles $\mathrm{ABC}$ and $\mathrm{CBD}$, we perform valence optimization by flipping the diagonal $\mathrm{BC}$ if and only if the quantity

$$
\sum_{p \in \triangle}(\operatorname{valence}(p)-6)^{2}
$$

is minimized after the flip. Since edge swaps can change the local topological properties of the mesh significantly when the mesh is coarse, this is only performed periodically after the number of vertices exceeds 500, or some other sufficiently large number. Furthermore, edge swapping can be very expensive; hence it is performed only when the average valence deviates too far from 6 . This is controlled by a parameter that can be altered dynamically by the user. Combining the three operations, we can achieve a provably good mesh. Since the edge ratio is at most 1:2, triangle sizes vary by at most a factor of 4 .

In short, after every iteration of the mesh propagation we invoke a mesh improvement step that consists of the following three stages: first, all the edges below a threshold are collapsed; second, all the edges above another threshold are bisected; finally, edge flipping is performed to improve aspect ratios of the existing triangles. For more details on the algorithm the reader is referred to. ${ }^{6}$

\subsection{Hard Constraints}

User specified constraint points can be added near ill-defined boundaries to help guide the segmentation. A moving triangle (all its vertices) will stop when it reaches the predefined neighborhood of the constraint point.

\subsection{Numerical Method}

We convert time derivatives into finite difference operators (in time) and solve the system by simple forward Euler integration.

$$
\mathbf{P}_{i}(\mathbf{r}, t+\triangle t)=\mathbf{P}_{i}(\mathbf{r}, t)+\left(\mathbf{F}\left(\mathbf{P}_{\mathbf{i}}\right)-\beta \cdot \overline{\mathcal{K}} \mathbf{n}(\mathbf{P})_{i}\right) \Delta t
$$

Time step $\triangle t$ and coefficients $\alpha, \beta$ and $\gamma$ are chosen empirically to provide convergence. Since tension and ballooning forces are defined directly on the surface, we do not need to discretize the volume to find the solution. The image force is also only computed at the $3 \mathrm{D}$ location where it acts on the vertices of the mesh.

After every time step, the mesh is updated using dynamic meshing (2.5), thus the number of vertices and connectivities are changing every iteration step. This step guarantees smooth and consistent properties of the mesh.

\section{IMPLEMENTATION AND RESULTS}

Our dynamic deformable model has been implemented in a software system written in C++ using the OpenGL, GLUT and GLUI libraries. It provides an interface for a user to tag key features in the data with constraint points and to control the model's deformation by interactively adjusting parameters. The data is presented on three orthogonal cutting planes, which may be individually swepted through the data. The constraint points may be placed directly on any of the three planes, completely specifying their location in Cartesian space.

In practice, orienting objects in 3D space is often challenging, especially when the resolution in one of the dimensions is low. Thus, we have also added a mechanism to enhance the selection of points, by adding windows with individual slices near the bottom of the interface. An arbitrary number of slices can be displayed in an array of embedded windows, whereby the user can select any of the slices and place constraint points. A 3D window is still maintained concurrently for the visualization of all the constraint points and the expanding mesh. Points can be added and removed in either window. See Figure 1. A control panel is used to adjust all parameters dynamically, while the program is running. For instance, the image force can be increased, once the dynamic model approaches a weak boundary. See Figure 2 
A GLUI interface and GLUT menus are available for easy navigation of the software system. Directional arrows can be dragged to move and resize the mesh as well as the orthogonal planes. Options for hiding the mesh, orthogonal planes, or constraint points are also readily accessible in the form of checkboxes. Different modes of rendering are kept in radio boxes, while parameters for expanding and subdividing the mesh can be dynamically changed in text boxes during the expansion phase. More advanced options for saving and loading constraint points and the mesh are available through GLUT menus.

Figures 3, 4 and 5 present segmentation results produced by our software system. Figure 3 contains a close-up of one of the cutting planes after a user has placed constraint points. In Figure 4 the initial model has begun to fit to the wall of the left ventricle. The mesh model is overlayed on the cutting plane. Figure 5 contains four models produced during the dynamic deformation process. The top left is the initial model. The top right and bottom left are intermediate shapes. The final model is presented in the bottom right.

\section{CONCLUSIONS}

We have presented a new dynamic deformable modeling approach to 3D segmentation. It utilizes recently developed dynamic remeshing techniques and curvature estimation methods to produce high-quality meshes. The approach has been implemented in an interactive environment that allows a user to specify an initial model and identify key features in the data. These features act as hard constraints that the model must not pass through as it deforms. We have employed the method to perform semi-automatic segmentation of heart structures from cine MRI data.

Future work will incorporate an implicit integration scheme to enhance the stability of the solution. Based on our preliminary work, we anticipate accurate segmentation will reduce postprocessing time by more than $50 \%$. This will allow more widespread use of cardiac function analysis in pediatric cardiovascular disease.

\section{REFERENCES}

1. I. Bankman, ed., Handbook of Medical Imaging, Academic Press, San Diego, CA, 2000.

2. A. Frangi, W. Niessen, and M. Viergever, "Three-dimensional modeling for functional analysis of cardiac images: A review," IEEE Trans. on Medical Imaging 20, pp. 2-25, January 2001.

3. A. Singh, D. Goldgof, and D. Terzopoulos, eds., Deformable Models in Medical Image Analysis, IEEE Computer Society, Los Alamitos, CA, 1998.

4. J. Miller, D. Breen, W. Lorensen, R. O'Bara, and M. Wozny, "Geometrically Deformed Models: A method for extracting closed geometric models from volume data," in Proc. SIGGRAPH '91, 25(4), pp. 217-226, 1991.

5. T. McInerney and D. Terzopoulos, "A dynamic finite element surface model for segmentation and tracking in multidimensional medical images with application to cardiac 4D image analysis," Computerized Medical Imaging and Graphics 19(1), pp. 69-83, 1995.

6. L. Kobbelt, T. Bareuther, and H.-P. Seidel, "Multiresolution shape deformations for meshes with dynamic vertex connectivity," in Proc. Eurographics 2000, p. C249, 2000.

7. M. Desbrun, M. Meyer, P. Schröder, and A. Barr, "Implicit fairing of irregular meshes using diffusion and curvature flow," in Proc. SIGGRAPH '99, pp. 317-324, 1999.

8. R. Malladi, J. Sethian, and B. Vemuri, "Shape modeling with front propagation: A level set approach," IEEE Trans. on Pattern Analysis and Machine Intelligence 17(2), pp. 158-175, 1995.

9. R. Whitaker, D. Breen, K. Museth, and N. Soni, "Segmentation of biological datasets using a level-set framework," in Volume Graphics 2001, M. Chen and A. Kaufman, eds., pp. 249-263, Springer, Vienna, 2001.

10. A. Gupta, T. O'Donnell, and A. Singh, "Segmentation and tracking of cine cardiac MR and CT images using a 3-D deformable model," in Proc. IEEE Conference on Computers in Cardiology, pp. 661-664, 1994. 


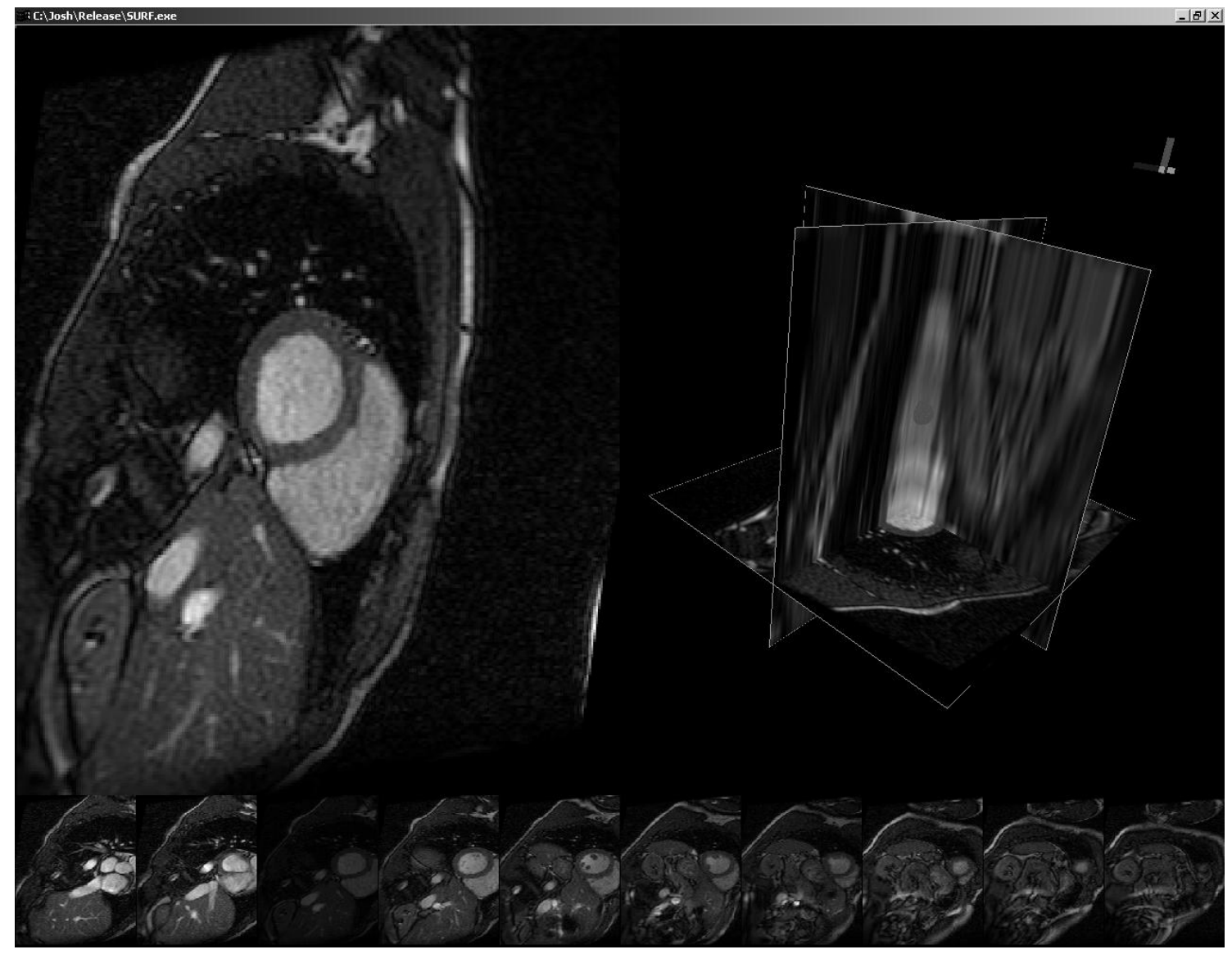

Figure 1: Main window of our software system displaying a loaded heart MRI dataset with several constraint points.

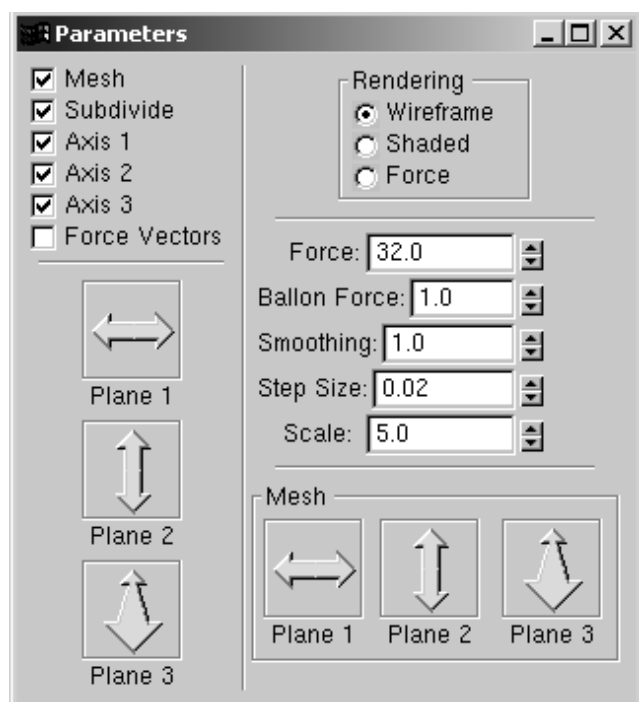

Figure 2: A control panel for setting model parameters. 


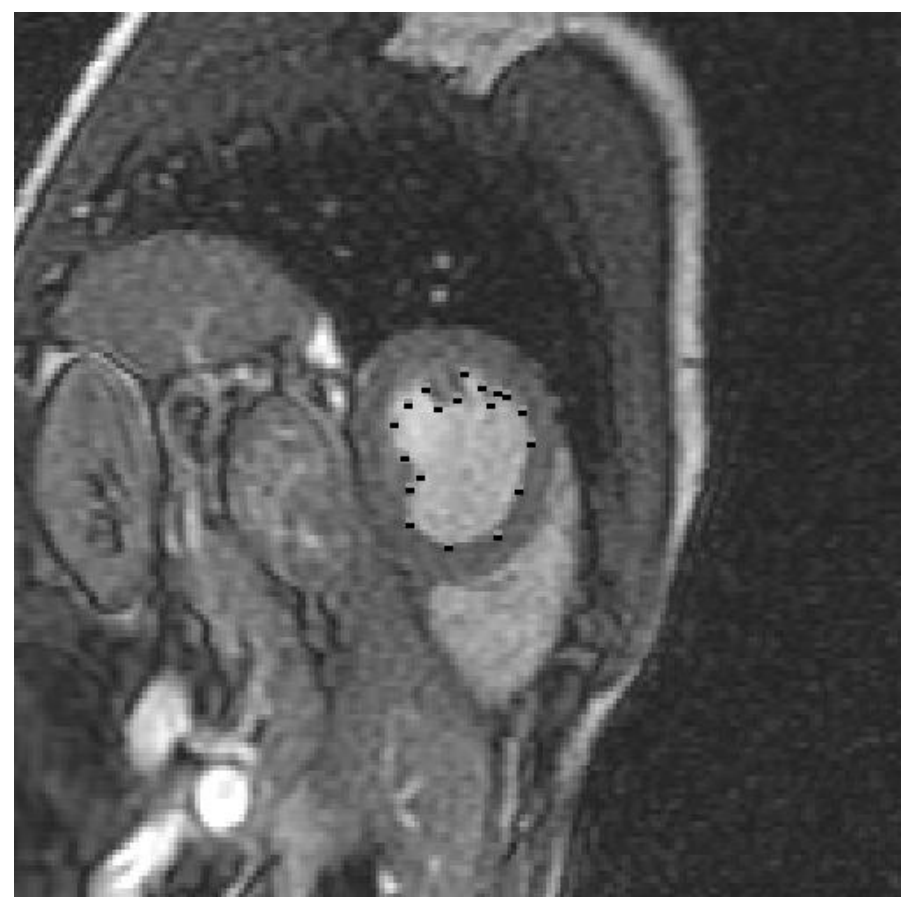

Figure 3: A close-up of a single slice of MRI data, showing user-defined constraint points.

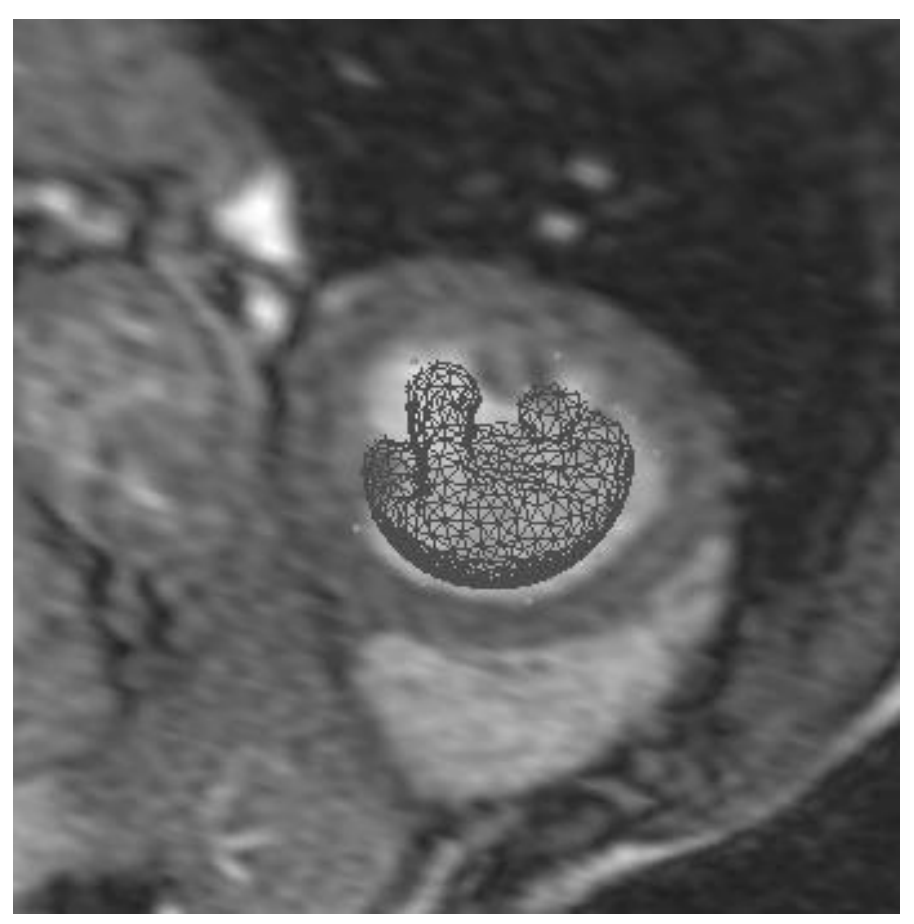

Figure 4: Evolving deformable model overlayed on a slice of MRI data. 

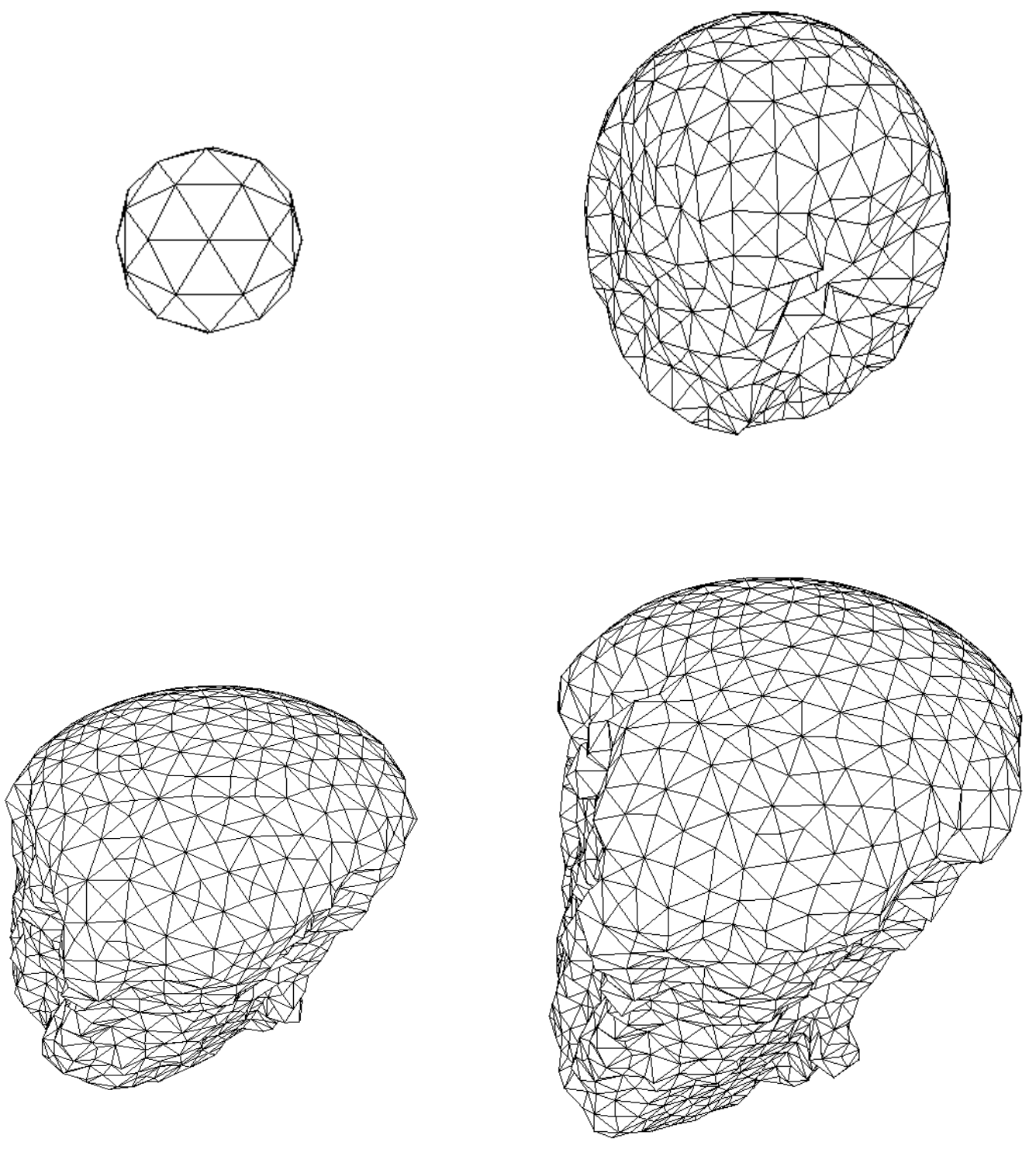

Figure 5: A sequence of dynamic models produced from a segmentation of the left ventricle. 